\title{
Propofol or Sevoflurane for Laryngeal Mask Airway Insertion-A Comparative Study
}

\author{
Authors \\ Dr Ushakumari.P.R ${ }^{1}$, Dr Harikumar.C.K ${ }^{2}$ \\ ${ }^{1}$ Additional Professor, Anaesthesiology, Medical College Trivandrum. \\ ${ }^{2}$ Assistant Professor, Department of Anaesthesiology, Medical College Trivandrum \\ *Corresponding Author \\ Dr Harikumar.C.K \\ Assistant Professor, Department of Anaesthesiology, Medical College Trivandrum
}

\begin{abstract}
Induction agents were proposed. This study attempts to compare effects of propofol and sevoflurane with the help of an accepted scoring system. We conducted this prospective study at SAT hospital government medical college Trivandrum after ethics committee clearance. The inclusion criteria were pediatric patients aged 3 to 12 years, of ASA grade, I and II, undergoing various surgical procedures such as such as herniotomy, circumcision, urethroplasty and lower limb surgeries. Exclusion criteria were those at increased risk of aspiration, with difficult airway, history of convulsions and ISL. This study had a sample size of 25 each patient selected randomly in the two groups namely propofol group and sevoflurane group. Details about basic demographic features and components of the scoring systems were collected in a pretested case report form. All statistical analyses were done in $R$ statistical environment. Study had more males compared to

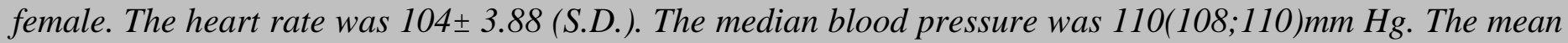
weight was 14(13;16). Total score for Propofol showed-poor 7 (28\%), satisfactory $15(60 \%)$ and excellent 3 (12\%). Regarding Sevoflurane, the scores were - poor 12 (48\%), satisfactory 9 (36\%) and excellent 4 (16\%). There was a statistically significant difference between the two groups when the total scores were compared ( $p$ value <0.05). The result of study showed that Propofol and Sevoflurane fulfill many of the characteristics of an ideal induction agent for LMA insertion in pediatric patients which include rapid smooth induction, smooth maintenance, minimum side effects, not much post operative complications and no cardiorespiratory side effects. Both 1\% Propofol and Sevoflurane are excellent agents as induction agents for the LMA insertion in paediatric patients. Considering the parameters in the scoring system, Propofol is superior to Sevoflurane.Thus, from this study the conclusion is that propofol is a better induction agent for LMA insertion in paediatric patients compared to Sevoflurane and results are coinciding with that of other studies.
\end{abstract}

\section{Introduction}

Maintenance of airway during induction of anesthesia and post induction for short procedures in pediatric patients was usually done by holding the mask and by maintaining spontaneous ventilation ${ }^{1}$. After the invention of LMA, it gained much importance for maintaining the airway during short procedures. Even though, various other airways maintaining devices have been introduced LMA only has gained much popularity $^{2-5}$.

During the past years many induction agents, both intravenous and inhalational agents have been advocated, which can be used for introduction of 
LMA. ${ }^{6,7}$ But no agent has been selected as an universal agent as each of them have its own adverse effects and other drawbacks.

For the introduction of LMA in adult as well as pediatric patient's certain anesthesia depth is needed. Intravenous and inhalational agents have been used in various doses and concentrations for the introduction of LMA. During the selection of an induction agent either intravenous and inhalational, it should have rapid and smooth induction, steady maintenance and smooth recovery.

An ideal induction agent for the introduction of LMA should possess the following characteristics.

1. It should provide smooth induction.

2. It should provide smooth maintenance.

3. It should have smooth recovery.

4. Minimum side effects.

5. It should maintain hemodynamic stability ${ }^{8}$. Recent reports have shown that both Sevoflurane and propofol fulfill many of these characteristics ${ }^{9}$. In this study, the responses to each drug are assessed by using a scoring system that are used for the ease of insertion of the LMA.

\section{Materials and Methods}

This is a prospective clinical comparative study was conducted at SAT Hospital, Medical College, Thiruvananthapuram after getting approval from the ethics committee. Informed consent was taken from parents for before conducting the study. All phases to the study were conducted in accordance with the declaration of the Helsinki.

Participants in this study included pediatric patients of ASA grade, I and II during the study period. We included children aged 3 to 12 years undergoing elective surgery with general anesthesia or regional anesthesiaduring surgical procedures such as herniotomy, circumcision, urethroplasty and lower limb surgeries. However, we excluded children at increased risk of aspiration and difficulty airway from this study. Moreover, Children with history of seizures and ISL were also not included in this study. We followed a random sampling strategy to enroll the patients. A prior sample size calculation showed required sample size as 25 each in both groups. Children were seen pre-operatively by the investigator. Detailed history with reference to history of congenital heart disease, drug allergy, aspiration chance, GERD, bronchial asthma, previous history of anesthesia, respiratory infection, obesity and difficult airway were taken. Clinical examination was done by the investigator. Blood routine results were examined and done routinely to all children. Children with any of the diseases noted and were excluded. There were two groups. Group 1 received propofol for induction and group 2 received Sevoflurane for induction.

Atropine $(0.02 \mathrm{mg} / \mathrm{kg}$ body weight $)$ and Midazolam $(0.02 \mathrm{mg} / \mathrm{kg}$ body weight $)$ was given before induction.

Prior to the induction of anesthesia, patients in both groups had a face mask placed over their face and was breathing spontaneously.

Group 1 (Propofol group) received intravenous Propofol (1.5 to $2.5 \mathrm{mg} / \mathrm{kg}$ body weight) with $100 \%$ oxygen via the face mask.

Group 2 (Sevoflurane group) children were induced with Sevoflurane 8\% in N20 and Oxygen. Jackson's rees circuit was primed with Sevoflurane $8 \%$ N20 and oxygen.

Loss of eyelash reflex was considered as the end point of induction in both groups. After the end point of induction, classical LMA insertion was attempted with appropriate size LMA.

Ease of introduction of classical LMA in each group was scored and graded as described in the literature $^{10}$. Scores below 16 were considered as poor, 16 to 17 were considered as satisfactory and 18 as excellent. The parameters noted were jawopening, ease for LMA insertion, coughing, gagging, laryngospasm and patient movement. All children were observed and scores were allotted by the investigator.

Principal investigator collected basic demographic and other relevant variables in a restructured and pretested case report form. Statistical analysis was done with $\mathrm{R}$ statistical software and Graphpad Instat software. Categorical data were summarized as frequencies and percentages and continuous 
data as mean and standard deviation or median and interquartile range. Mann-Whitney test was used to comparethe groups. Chi-square test was used to compare graded scores between the two groups.

\section{Results}

In the present study, there were 50 participants. All patients completed the study with no dropout or any missing values. These patients were equally divided to two groups, group I being the propofol group and group II sevoflurane group. The median age was $4(3 ; 5)$. There was a male predominance with $31(62 \%)$. The mean heart rate was $104 \pm 3.88$ (S.D.). The median blood pressure was $110(108 ; 110) \mathrm{mm} \mathrm{Hg}$. The median weight was 14(13;16).

All baseline characteristics in the study population across the two groups were comparable except heart rate(table1 and figure 1).

Table 1: Baselinecomparison between the groups.

\begin{tabular}{lcccc} 
& {$[$ ALL] N=50 } & propafol N=25 & sevoflurane N=25 & p.overall \\
\hline Age & $4.00[3.00 ; 5.00]$ & $4.00[3.00 ; 6.00]$ & $4.00[3.00 ; 5.00]$ & 0.216 \\
Sex: & $31(62.0 \%)$ & $14(56.0 \%)$ & $17(68.0 \%)$ & \\
male & $19(38.0 \%)$ & $11(44.0 \%)$ & $8(32.0 \%)$ & \\
female & $104(3.88)$ & $102(3.77)$ & $106(2.87)$ & $<0.001$ \\
Heart rate & $110[108 ; 110]$ & $110[104 ; 110]$ & $110[108 ; 110]$ & 0.602 \\
Mean Blood.Pressure & $14.0[13.2 ; 16.0]$ & $15.0[14.0 ; 18.0]$ & $14.0[12.0 ; 16.0]$ & 0.093 \\
Weight & & & & 0.495 \\
ASA: & $39(78.0 \%)$ & $18(72.0 \%)$ & $21(84.0 \%)$ & \\
1 & $11(22.0 \%)$ & $7(28.0 \%)$ & $4(16.0 \%)$ &
\end{tabular}

Figure 1: comparison of induction time between the two groups

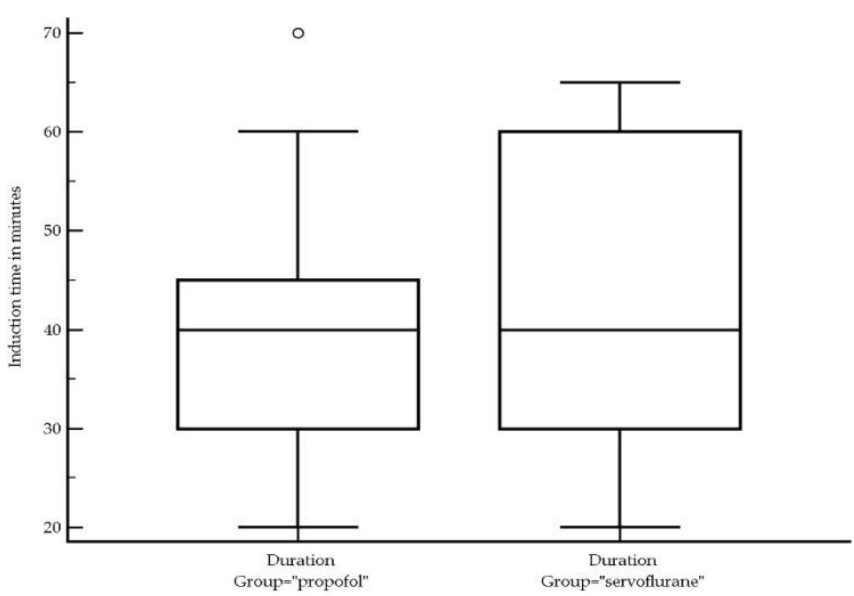

In jaw opening, for partial it is $14(56 \%)$ for Propofol and 15 (60\%) for Sevoflurane, for full it is $11(44 \%)$ for Propofol and 10 (40\%) for Sevoflurane. There was no statistically significant difference in the jaw opening between the two groups $(\mathrm{p}$ value>0.05). Inease of insertion, for difficult it is 7 (28\%) for Propofol and 8 (32\%) for Sevoflurane, for easy it is $18(72 \%)$ for Propofol and $17(68 \%)$ for Sevoflurane. There was no statically significant difference between the two groups ( $p$ value $>0.05$ ). Incoughing, for minor it is
1 (4\%) for Propofol and 0 (0\%) for Sevoflurane, for nil it is 24 (96\%) for Propofol and 25 (100\%) for Sevoflurane. There was no statistically significant difference between the two groups. In gagging, for minor it is $4(16 \%)$ for Propofol and $2(8 \%)$ for Sevoflurane, for nil it is $21(84 \%)$ for Propofol and $23(92 \%)$ for Sevoflurane, which was not statically significant. In Laryngospasm, for partial it is 5 (20\%) for Propofol and 10 (40\%) for Sevoflurane, for nil it is 20 (80\%) for Propofol and $15(60 \%)$ for Sevoflurane, which failed to reach statistical significance between the groups. In patient movement, for moderate it is $15(60 \%)$ for Propofol and 19 (76\%) for Sevoflurane, for nil it is $10(40 \%)$ for Propofol and $6(24 \%)$ for Sevoflurane. This relation also showed no statistically significant association.In total score regarding Propofol - the scores were poor 7 (28\%), satisfactory $15(60 \%)$ and excellent 3 $(12 \%)$. Regarding Sevoflurane - the scores were poor $12(48 \%)$, satisfactory $9(36 \%)$ and excellent $4(16 \%)$. There was a statistically significant difference between the two groups when the total scores were compared(p value <0.05)(table 2). 
Table 2: comparison of various parameters between Propofol and sevoflurane groups

\begin{tabular}{lcccc} 
& [ALL] N=50 & propofol N=25 & sevoflurane N=25 & p.overall \\
\hline Jaw Opening: & & & & 1.000 \\
$\quad$ full & $21(42.0 \%)$ & $11(44.0 \%)$ & $10(40.0 \%)$ & \\
$\quad$ partial & $29(58.0 \%)$ & $14(56.0 \%)$ & $15(60.0 \%)$ & \\
Ease of insertion: & & & & 1.000 \\
$\quad$ difficult & $15(30.0 \%)$ & $7(28.0 \%)$ & $8(32.0 \%)$ & \\
$\quad$ easy & $34(68.0 \%)$ & $17(68.0 \%)$ & $17(68.0 \%)$ & \\
NA & $1(2.00 \%)$ & $1(4.00 \%)$ & $0(0.00 \%)$ & \\
Coughing: & $1(2.00 \%)$ & $1(4.00 \%)$ & $0(0.00 \%)$ & 1.000 \\
$\quad$ minor & $49(98.0 \%)$ & $24(96.0 \%)$ & $25(100 \%)$ & \\
$\quad$ nil & & & & \\
Gagging: & $6(12.0 \%)$ & $4(16.0 \%)$ & $2(8.00 \%)$ & \\
$\quad$ minor & $44(88.0 \%)$ & $21(84.0 \%)$ & $23(92.0 \%)$ & \\
$\quad$ nil & & & & \\
Laryngospasm: & $35(70.0 \%)$ & $20(80.0 \%)$ & $15(60.0 \%)$ & \\
$\quad$ nil & $15(30.0 \%)$ & $5(20.0 \%)$ & $10(40.0 \%)$ & \\
$\quad$ partial & & & & \\
Patient Movement: & & & \\
$\quad$ moderate & $34(68.0 \%)$ & $15(60.0 \%)$ & $19(76.0 \%)$ & \\
$\quad$ nil & $16(32.0 \%)$ & $10(40.0 \%)$ & $6(24.0 \%)$ &
\end{tabular}

\section{Discussion}

The purpose of this study was to compare the ease of insertion of LMA with propofol and sevoflurane.

Intubating conditions for the Laryngeal Mask Airway using Propofol and Sevoflurane were compared favorably two groups. It is found that for the same end point of induction, which is loss of eye lash reflex in both the groups, conditions for Laryngeal Mask Airway insertion were superior with Propofol than with Sevoflurane. The laryngeal Mask airway could be successfully placed both the groups. However, favourable conditions for insertion of the LMA were seen in more with Propofol. This was probably due to the inadequate jaw relaxation with sevoflurane. Propofol is known to have a relaxant effect on jaw muscles whereas inhalational anaesthetics may cause an increased muscle tone and spasticity. Therefore, for a similar end point of induction, i.e. loss of eye lash reflex, there may be greater jaw relaxation with Propofol. These findings are in consistent with the findings in literature ${ }^{11}$

Laryngeal Mask Airway placement requires suppression of the less sensitive hypopharynx for successful placement as well as attenuation of the laryngeal reflexes in order to reduce stimulation of the anterior laryngeal structures during insertion. Propofol is known to depress laryngeal reflexes, thus facilitating Laryngeal Mask Insertion. This feature explains the less incidence of laryngospasm in the Propofol group. Other features like coughing, gagging and patient movement did not reach statistical significance. The haemodynamic responses were stable for both the groups. Study by $\mathrm{Li}$ et al supports these findings 12,13

Attempts were made to see whether the two groups were identical with respect to age, gender, ASA grading and diagnosis. Attempts were made to see the response of two drugs for LMA insertion by using the scoring system.

Considering total score, Propofol is superior to Sevoflurane as induction agent for LMA insertion in pediatric patients, as Propofol is having higher scores.

The result of study showed that Propofol and Sevoflurane fulfill many of the characteristics of an ideal induction agent for LMA insertion in pediatric patients which include rapid smooth induction, smooth maintenance, minimum side effects, not much post operative complications and no cardiorespiratory side effects. Both $1 \%$ Propofol and Sevoflurane are excellent agents as induction agents for the LMA insertion in pediatric patients. Considering the parameters in the scoring system, Propofol is superior to Sevoflurane.

Thus, from this study the conclusion is that propofol is a better induction agent for LMA 
insertion in pediatric patients compared to Sevoflurane, and results are coinciding with that of other studies.

Comparing the groups, Propofol can be used safely for pediatric LMA insertion and though Sevoflurane is a good induction agent with good recovery, considering safety features, Propofol comes as better drug in my study with minimum side effects. Since our sample size was apt for our analysis, we got a comparable and consistent result with other similar publications.

\section{References}

1. Harless J, Ramaiah R, Bhananker SM. Pediatric airway management. International Journal of Critical Illness and Injury Science 2014; 4(1): 65-70.

2. Chang T-L. Laryngeal mask airway. Google Patents; 2004.

3. Pagan E. Laryngeal mask airways. Google Patents; 1998.

4. Finucane BT, Tsui BC, Santora AH. The difficult airway. Principles of airway management 2011: 361-414.

5. Baskett P, Bossaert L, Carli P, et al. Guidelines for the advanced management of the airway and ventilation during resuscitation: a statement by the Airway and Ventilation Management Working Group of the European Resuscitation Council. Resuscitation 1996; 31(3): 20130.

6. Zielinska M, Holtby H, Wolf A. Pro-con debate: intravenous vs inhalation induction of anesthesia in children. Pediatric Anesthesia 2011; 21(2): 159-68.

7. Brambrink AM, Braun U. Airway management in infants and children. Best Practice \& Research Clinical Anaesthesiology 2005; 19(4): 675-97.

8. Eger I, Edmond I. Characteristics of anesthetic agents used for induction and maintenance of general anesthesia. American Journal of Health-System Pharmacy 2004; 61.
9. Chowdhury T, Prabhakar H, Bharati SJ, Goyal K, Dube SK, Singh GP. Comparison of propofol versus sevoflurane on thermoregulation in patients undergoing transsphenoidal pituitary surgery: A preliminary study. Saudi J Anaesth 2012; 6(1): 12-5.

10. Priya V, Divatia J, Dasgupta D. A comparison of propofol versus sevoflurane for laryngeal mask airway insertion. Indian J Anaesth 2002; 46(1): 31-4.

11. Uezono $\mathrm{S}$, Goto $\mathrm{T}$, Terui $\mathrm{K}$, et al. Emergence agitation after sevoflurane versus propofol in pediatric patients. Anesth Analg 2000; 91(3): 563-6.

12. Li J, Ye LS, Gao P, et al. [Optimal concentrations of sevoflurane and propofol for laryngeal mask airway insertion in Chinese boys of different ages]. Zhonghua Yi Xue Za Zhi 2009; 89(15): 1012-5.

13. Oberer C, von Ungern-Sternberg BS, Frei FJ, Erb TO. Respiratory reflex responses of the larynx differ between sevoflurane and propofol in pediatric patients. Anesthesiology 2005; 103(6): 1142-8. 